\title{
Correction to: Discovery of the RANKL/RANK/OPG system
}

\author{
Hisataka Yasuda ${ }^{1} \mathbb{C}$
}

Published online: 13 January 2021

(c) The Japanese Society Bone and Mineral Research 2021

\section{Correction to: Journal of Bone and Mineral Metabolism https://doi.org/10.1007/s00774-020-01175-1}

In the original publication of the article, reference 12 was published incorrectly as follows, "Mizuno A, Amizuka N, Irie K, Murakami A, Fujise N, Kanno T, Sato Y, Nakagawa N, Yasuda H, Mochizuki S, Gomibuchi T, Yano K, Shima N, Washida N, Tsuda E, MorinagaT HK, Ozawa H (1998) Severe osteoporosis in mice lacking osteoclastogenesis inhibitory factor/osteoprotegerin. Biochem Biophys Res Commun 247:610-615".

The correct reference is provided in this correction.

12. Mizuno A, Amizuka N, Irie K, Murakami A, Fujise N, Kanno T, Sato Y, Nakagawa N, Yasuda H, Mochizuki S, Gomibuchi T, Yano K, Shima N, Washida N, Tsuda E,
MorinagaT, Higashio K, Ozawa H (1998) Severe osteoporosis in mice lacking osteoclastogenesis inhibitory factor/osteoprotegerin. Biochem Biophys Res Commun 247:610-615

Publisher's Note Springer Nature remains neutral with regard to jurisdictional claims in published maps and institutional affiliations.

The original article can be found online at https://doi.org/10.1007/ s00774-020-01175-1.

Hisataka Yasuda

yasuda.hisataka@nisshin.com

1 Nagahama Institute for Biochemical Science, Oriental Yeast

Co., Ltd., 50, Kano-cho, Nagahama, Shiga 526-0804, Japan 